\title{
Towards 3D printing of a monocoque transtibial prosthesis using a bio-inspired design workflow
}

\author{
Luca Gabriele De Vivo Nicoloso \\ Department of Structural Engineering, University of California San Diego, La Jolla, California, USA \\ Joshua Pelz \\ Department of Mechanical and Aerospace Engineering, University of California San Diego, La Jolla, California, USA \\ Herb Barrack \\ LIMBER Prosthetic and Orthotics Inc, San Diego, California, USA, and \\ Falko Kuester \\ Department of Structural Engineering, University of California San Diego, La Jolla, California, USA
}

\begin{abstract}
Purpose - There are over 40 million amputees globally with more than 185,000 Americans losing their limbs every year. For most of the world, prosthetic devices remain too expensive and uncomfortable. This paper aims to outline advancements made by a multidisciplinary research group, interested in advancing the restoration of human motion through accessible lower limb prostheses.

Design/methodology/approach - Customization, comfort and functionality are the most important metrics reported by prosthetists and patients. The work of this paper presents the design and manufacturing of a custom made, cost-effective and functional three-dimensional (3D) printed transtibial prosthesis monocoque design. The design of the prosthesis integrates 3D imaging, modelling and optimization techniques coupled with additive manufacturing.

Findings - The successful fabrication of a functional monocoque prosthesis through 3D printing indicates the workflow may be a solution to the worldwide accessibility crisis. The digital workflow developed in this work offers great potential for providing prosthetic devices to rural communities, which lack access to skilled prosthetic physicians. The authors found that using the workflow together with 3D printing, this study can create custom monocoque prostheses (Figure 16). These prostheses are comfortable, functional and properly aligned. In comparison with traditional prosthetic devices, the authors slowered the average cost, weight and time of production by $95 \%, 55 \%$ and $95 \%$, respectively.

Social implications - This novel digital design and manufacturing workflow has the potential to democratize and globally proliferate access to prosthetic devices, which restore the patient's mobility, quality of life and health. LIMBER's toolbox can reach places where proper prosthetic and orthotic care is not available. The digital workflow reduces the cost of making custom devices by an order of magnitude, enabling broader reach, faster access and improved comfort. This is particularly important for children who grow quickly and need new devices every few months or years, timely access is both physically and psychologically important.

Originality/value - In this manuscript, the authors show the application of digital design techniques for fabricating prosthetic devices. The proposed workflow implements several advantageous changes and, most importantly, digitally blends the three components of a transtibial prosthesis into a single, 3D printable monocoque device. The development of a novel unibody transtibial device that is properly aligned and adjusted digitally, greatly reduces the number of visits an amputee must make to a clinic to have a certified prosthetist adjust and modify their prosthesis. The authors believe this novel workflow has the potential to ease the worldwide accessibility crisis for prostheses.
\end{abstract}

Keywords Polymers, Additive manufacturing, Prostheses, 3D imaging

Paper type Research paper

\section{Abbreviations}

The following abbreviations are used in this manuscript:

$$
\begin{aligned}
& \text { WHO }=\text { World Health Organization; } \\
& \text { CFRP }=\text { Carbon Fibre Reinforced Polymer; } \\
& \text { TKA }=\text { Trochanter, Knee and Ankle; }
\end{aligned}
$$

The current issue and full text archive of this journal is available on Emerald Insight at: https://www.emerald.com/insight/1355-2546.htm

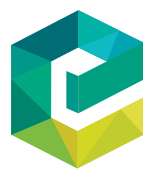

Rapid Prototyping Journal

27/11 (2021) 67-80

Emerald Publishing Limited [ISSN 1355-2546]

[DOI 10.1108/RPJ-06-2021-0136]
PTB = Patellar Tendon Bearing;

WWII = World War Two;

CAD = Computer-Aided Design;

VASS = Vacuum-Assisted Suspension System;

(C) Luca Gabriele De Vivo Nicoloso, Joshua Pelz, Herb Barrack and Falko Kuester. Published by Emerald Publishing Limited. This article is published under the Creative Commons Attribution (CC BY 4.0) licence. Anyone may reproduce, distribute, translate and create derivative works of this article (for both commercial and non-commercial purposes), subject to full attribution to the original publication and authors. The full terms of this licence may be seen at http://creativecommons.org/licences/by/4.0/legalcode

Received 9 June 2021

Revised 6 July 2021

Accepted 6 July 2021 


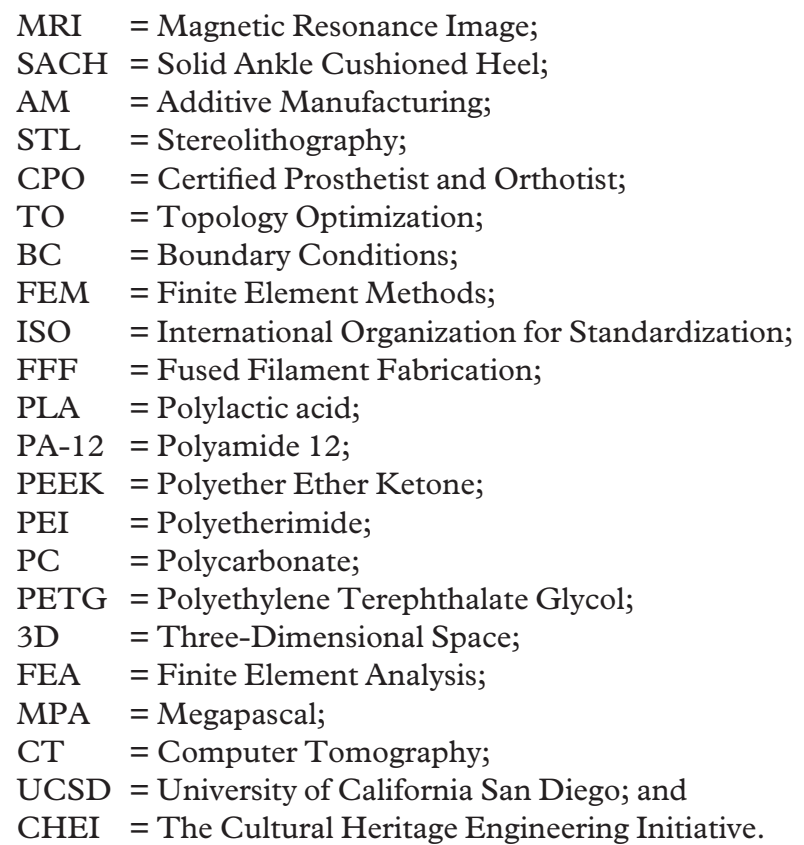

\section{Introduction: global challenge: access to prosthetic devices}

The World Health Organization (WHO) estimates that there are 40 million amputees throughout the developing world, but only about $5 \%$ of them have access to any form of prosthetic devices or assistance (Schuch and Pritham, 1994). The result? A staggering 38 million people worldwide, 2 million of whom are in the USA, live painful and restrictive lives. Vascular disease $(54 \%)$ - often in the form of diabetes and peripheral arterial disease - trauma ( $45 \%$ ) and cancer (less than $2 \%$ ) cause more than 185,000 Americans to lose limbs every year (ZieglerGraham et al., 2008). Millions of amputees are left behind because prosthetics remain too expensive and uncomfortable. Custom-fit requirements make it difficult to mass-produce lowcost devices and the lack of access to proper health care and medical professionals prevents adjustments needed to maintain safe, comfortable prosthetic devices. Imperfections at the prosthetic-limb interface can be the difference between an amputee wearing their prosthesis or choosing to sacrifice access to the world we take for granted. This study was profoundly motivated to develop devices that could realistically satisfy the current and future global demand. Current prosthetic sector challenges were summarized in the paragraph below (Schuch and Pritham, 1994):

Prosthetic devices are frequently not included in national health and social insurance systems, leading to limited funding. There is a shortage of trained clinicians who can make safe, custom-fit prosthetic and orthopaedic mobility devices. The WHO estimates 40,000 more clinicians are needed to meet demand. There is limited availability of appropriate products in many countries. The traditional methods of producing customfit devices are expensive and can take days to weeks. Patients must attend many appointments and often need to travel long distances at their own expense. Services for the poor are usually provided by charities, which results in low-quality control and potentially unsafe prostheses.

\subsection{The state of knowledge: transtibial prostheses}

There are three necessary components to a transtibial prosthesis: the socket, pylon and ankle-foot complex (Figure 1). The socket attaches the prosthesis to the wearer; this attachment can be through means of corsets, straps, lock pins or vacuums. Sockets are mostly made from composite materials such as carbon fibre reinforced polymer (CFRP). The pylon, commonly made of medical-grade metals, is used to adjust the height and transfer load between the socket and foot. These three components are usually attached with pyramid connectors, which allow for proper trochanter(hip)-knee-ankle (TKA) line adjustment. The ankle-foot complex serves to interface with the ground and can be active or passive, with the goal of enabling a proper gait cycle or walking motion.

The modern era for prosthetics started shortly after Second World War with the introduction of the patellar tendon bearing (PTB) socket (LeMoyne, 2016). The PTB socket capitalizes on the pressure tolerant areas of the residual limb such as the tendons and flat structures of bone, to support the forces necessary to restore ambulation. This attachment to the wearer is the primary function of the socket. In recent years, socket science and engineering have dramatically benefited from advancements in modern medical technology. Modelling of the residual limb to custom fit the socket is being done via direct surface scanning and integrated with magnetic resonance image (MRI) data to map bone tissue depth to socket material stiffness properties (Sengeh and Herr, 2013). Osseointegrated prosthetic sockets use attachment points embedded in the residual bone, a method recently approved by the US Food and Drug Administration (Al Muderis et al., 2017). Computeraided design (CAD) software is being used to manufacture moulds of the residual limb, over which thermoplastic materials can be shaped and formed to the user's specific needs (Schall and Slemker, 1998). These plastic sockets can be adjusted via thermoforming multiple times during the early amputation healing stages as changes occur in the residual limb.

To obtain a well-fitted socket, patients must currently undergo several rounds of appointments with certified prosthetists. These experts take measurements of the residual limb with a fitted liner and then mark anatomical areas on the limb. After assessing the limb, the prosthetist will use plaster bandages to create a cast around the limb. The anatomical marks will transfer to the interior of the mould, such that the prosthetist can attempt to design the socket to consider regions of bone and soft tissue. The prosthetist can manipulate the

Figure 1 Typical pylon with socket and foot attached

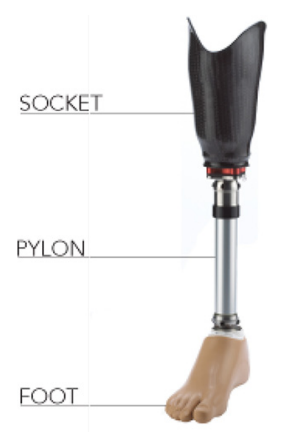


plaster bandages whilst they are hardening to adjust their shape. This shaping requires years of experience and will only result in a comfortable, functional socket if the prosthetist is highly skilled. Once the plaster hardens, the cast is removed and used to create a positive plastic mould that experts can build off of to create a check socket.

The marking of anatomical landmarks on the residual limb is essential because some regions of the limb, like bony protrusions, must be avoided to prevent patient discomfort, whilst other areas such as flat bones and soft tissue can bear loads safely. Prosthetists must ensure proper distribution of pressure to avoid hot spots, which cause discomfort and led to health complications. For transtibial amputees, it is essential to avoid placing strain on the tibia (shinbone) and fibular nerves. Instead, the load should be focused along the patellar tendon and the soft tissue along the sides of the shin bone. It is also essential to design a socket that enables the natural hinging movement of the knee. The difficult nature of socket design is a major contributor to the accessibility crisis because the majority of amputees do not have access to skilled specialists who can properly make these patient-specific adjustments.

Modern socket design often incorporates a liner in between the patient's skin and socket to increase comfort and provide energy absorption. These sock-like devices, frequently made of silicon, can help prevent "pistoning" (movement of the residual limb within the socket) when used in conjunction with a vacuum check valve or vacuum-assisted suspension system. Unfortunately, the silicon liners are also susceptible to the transmission of shear forces that can create friction-based "hotspots" on the residual limb (LeMoyne, 2016). Numerous studies have been conducted on the designs of sockets, insocket interface pressure measurements and socket biomechanics, all of which reiterate the importance of the socket in the overall design of the prosthetic device (Pirouzi et al., 2014). One clear example is the work done at the Massachusetts Institute of Technology media lab by $\mathrm{PhD}$ student David Sengeh and Professor Hugh Herr. Their research focuses on variable impedance sockets designed from MRI data. The idea is to use a computer algorithm to assign regions of variable material stiffness on the socket walls based on information gathered from MRI scans and then use a multimaterial three-dimensional (3D) printer to allocate different materials where designed. The authors claim to have reduced the pressure experienced by the wearer by up to $17 \%$ (Sengeh and Herr, 2013). As a result of these academic efforts, companies such as ProsFit and Mercuris, are using multimaterial 3D printing for manufacturing custom-fit sockets. ProsFit (Bulgaria) and Mercuris (Germany) have teamed up to produce a low-cost transtibial prosthesis for trial in the United Arab Emirates. Although the device is well beyond the price targets for developing nations, the proof-of-concept and materials testing experience it provides is invaluable.

Attached to the socket is the pylon, which controls load transfer to the ankle-foot complex and, in some cases, provides energy recapture during the gait cycle. Typically constructed of stainless steel, titanium or aluminum, the pylon can be extendable to accommodate for growth and may also contain a shock absorption system to facilitate ambulation. Interestingly, the pylon section of pre-second world war prostheses in Japan was made exclusively out of bamboo, a practice that was abandoned as the country became more globally integrated (LeMoyne, 2016). Recently, however, bamboo is being studied as a subject of bioinspiration for pylon design due to its structural hierarchy, which enables strength and stiffness at low weight (Strait, 2006).

The ankle-foot complex is the prosthetic device's interface to the ground and its design, to a large degree, determines the quality of an amputee's gait cycle. The human foot is a complicated system made up of over 20 bones and 30 joints. The structure of the ankle and foot offer necessary degrees of freedom - the expected range of motion changes with age, gender, body size and flexibility. Dorsiflexion, described as the lifting of the toes, can range from 10-20 degrees. Plantar flexion, described as the lowering of the toes, can range from 40-55 degrees. Inversion, described as curling your toes inwards, is estimated at 23 degrees. Finally, eversion is estimated at 12 degrees. The ankle-foot complex should replicate these ranges of motion to promote a correct gait cycle. The gait cycle begins with the heel strike, your toes curl up, then flatten out and finally push off before the swing phase. This motion is pivotal for activities involving jumping and landing. Landing flat-footed can be extremely painful as that force is driven directly into the knee joint, whereas landing on extended toes absorbs shock. That potential energy is converted into large displacements in a natural movement. Thus, shock absorption and energy return mechanisms separate high-performance devices from cheaper, passive devices.

In general, whether the foot design is passive or active, the top of the complex includes a horizontal edge to offer a connection with the pylon. The most common passive anklefoot complex is the solid ankle cushioned heel (SACH) model, which is a jointless prosthetic foot with a foam insert to enable a small amount of deflection. The limited range of motion inherent to the SACH foot results in asymmetric gait characteristics, high metabolic costs and low energy return benefits (LeMoyne, 2016). Whilst basic prosthetic foot models similar to the SACH model are still in use such as the Jaipur foot and the EB1 foot that is made with locally available materials, many other new materials and designs are emerging (Strait, 2006). These new models include carbon fibre bladetype feet, adapted mostly for running and the Niagara foot, a futuristic-looking stable ankle-foot made from Hytel. Hytel is a thermoplastic polyester elastomer that provides superior durability and energy return. The Niagara foot has been tested on more than 3,000,000 loading cycles and shows increased durability over carbon fibre feet. Seattle feet, which are numerous in design, incorporate moulded and manufactured components of various materials including carbon fibre, nylon and metal. Seattle feet can either be bolted directly to the pylon or attached with special adapters that simulate ankle articulation.

\subsection{Advanced manufacturing techniques}

3D imaging and additive manufacturing (AM), commonly referred to as "3D printing", are critical enablers for this study. AM enables complex geometries that are impossible to produce through traditional manufacturing techniques with the added benefit of quicker design and production times. The complexity and customization of parts do not increase processing time or 
cost, which enhances the applicability to prosthetic devices that must be custom-fit to every patient. Further, combining CAD with 3D imaging techniques speeds up the design process for patient-specific design features and because of the digital workflow, can be done simultaneously by several people around the world. Summarized below are what we think the most significant benefits of applying 3D imaging and 3D printing technologies into prostheses:

3D printing allows for faster production time: The time it takes to print a custom prosthetic is significantly reduced when compared with conventional manufacturing methods such as casting. Faster production time means we can deliver more devices in a shorter period, taking us a step closer to our goal of providing these devices to those unaddressed 38 million amputees around the world. Custom fitting comfort: Custom devices means the wearer does not have to worry anymore about the prosthetic being too big or small or just too uncomfortable. This translates into more satisfied patients who will use the devices to improve their lifestyle and that of their family members. Fewer clinic visits: A more efficient, digital production process means fewer trips to the clinic. The method of digital design allows a certified prosthetist to do most of the work virtually and without the need for a patient to be in the same room. The patient only needs to be physically present at a location for image data acquisition and for a fitting session at the end of the design and manufacturing process. Shorter hospital stays: Patients could be fitted and discharged with no or only one overnight stay. This will significantly reduce the cost of acquiring a prosthetic device, which we believe will improve the chances of patients receiving and wearing their prosthetics. Safe work environment: The technology we use is reliable for technicians and professional doctors to use and operate. Besides a scanner device and a 3D printer, the only tools needed are a computer and a set of software to process, design and manufacture a full wearable prosthetic. Develops digital skills: Clinicians will build expertise and skills in the latest 3D technology.

Whilst the application of digital design techniques for fabricating prosthetic devices has been previously demonstrated, our proposed workflow implements several advantageous changes and, most importantly, digitally blends the three components of a transtibial prosthesis into a single, 3D printable monocoque device. The development of a monocoque transtibial device that is properly aligned and adjusted digitally, greatly reduces the number of visits an amputee must make to a clinic to have a certified prosthetist adjust and modify their prosthesis.

\section{Materials and methods}

\subsection{Three-dimensional imaging and modelling}

One of the critical goals of this study was to allow for the costeffective 3D modelling of a patient's damaged limb through the use of smartphones and photogrammetry techniques. To confirm and quantify the quality and accuracy of the photogrammetry models, we imaged the patient's residual limb with photogrammetry techniques and with an industrystandard 3D structured light scanner. In addition to this, we scanned the waist and sound leg of the patient, which were used later during the component alignment process. This was a significant enabler for achieving a fully 3D printable monocoque, as we will discuss later. For photogrammetry, we selected a Sony a7iii camera (Sony, Japan) as our data acquisition unit and Metashape (Agisoft, Russia) as the photogrammetric processing software. For structured light scanning, we used the Omega 3D Scanner (Willow Wood, OH) and its companion Omega software package (Willow Wood, $\mathrm{OH})$. The OMEGA Scanner 3D has an acquisition frequency of 550,000 measurements/second and scanned geometries generated in real-time in the OMEGA software.

The residual limb scanning processes are shown in Figures 2 and 3. Firstly, the patient was placed in a standing position with a liner covering the residual limb. The liner helped us achieve an even lighting surface for improved imaging and capturing the real leg geometry that would be hosted by the socket. For the laser scanning process, we made sure a proper distance of approximately 15 inches was specified by the Omega 3D Scanner user manual, we covered all the regions of interest. We scan using a top to bottom movement followed by a rotational movement around the target limb followed by a bottom to top movement and so on and collect the necessary data for a $3 \mathrm{D}$ point cloud reconstruction. In a similar approach, following best photogrammetry practices (Bregler et al., 2000), we took 60 pictures manually at approximately 20 inches from the target and all around the residual limb, making sure to capture every section of the limb in multiple pictures to ensure feature overlapping. Photogrammetry works best when images are diverse and cover the target object from multiple angles and points of view. This allowed us to achieve proper resolution and accuracy without the need for a fixed rig around the patient's limb. Overlap between different pictures is important for the photogrammetry technique to work properly. Also, calibrated scale bars are critically important when trying to obtain accurate physical reconstruction and were provided by Agisoft. We placed three scale bars near the patient's limb using a flat surface parallel to the length of the patient limb as seen in Figure 2(b). Pictures were captured in . jpeg format using an iPhone 11 Pro Max (Apple, CA). Metashape was used to align each image and, using the calibrated scale bars, to scale the images to real dimensions. This process

\section{Figure 2 Photogrammetry workflow}
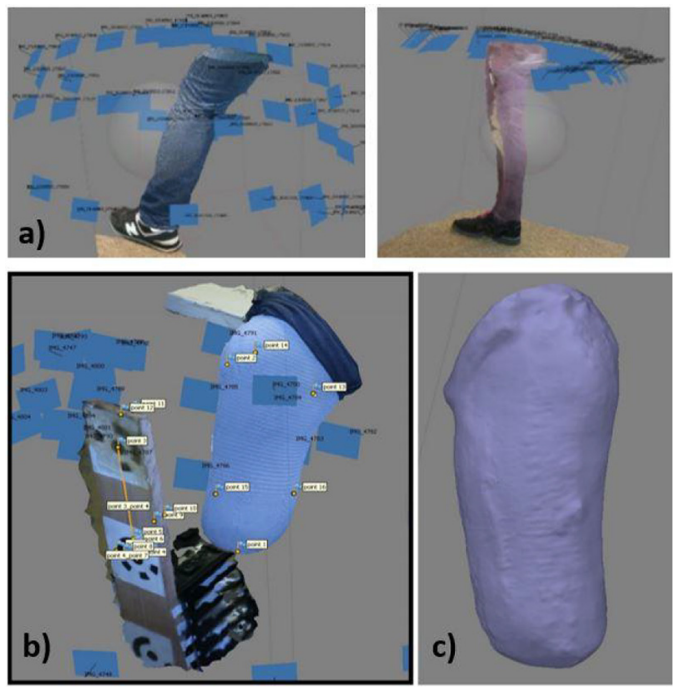
Figure 3 Laser scanning workflow
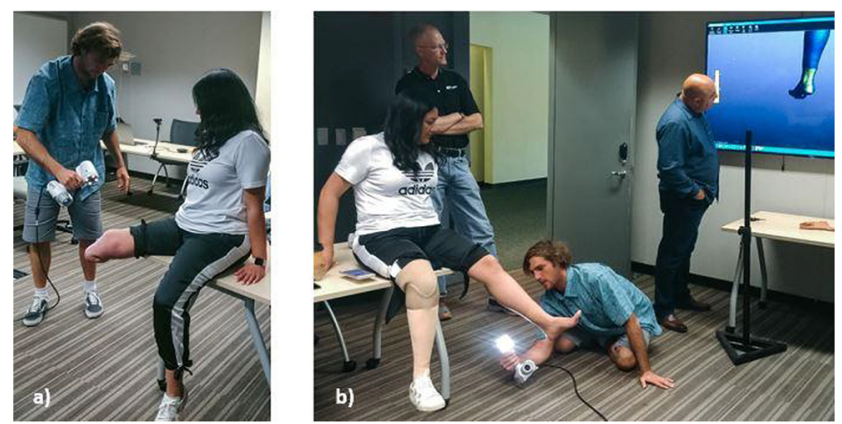

enabled the sparse and dense point clouds to be reconstructed. Finally, a mesh was built from the point clouds and exported in the stereolithography (STL) format.

In addition, an accurate model of the healthy leg was captured, which served as the reference geometry for the pylon and enabled alignment of the three components (socket, pylon and ankle-foot complex). Using the same approach previously described, a mesh model of the sound leg was generated. Subsequent processing steps for the sound leg model requires the conversion of this mesh to a solid body. This was performed using the CAD software Rhinoceros 3D (McNeel, WA), which allowed us to capture specific regions of the sound leg as solid bodies. The mirrored leg model was used to accurately size and align components and facilitated the design of the monocoque device, displayed in Figure 4.

\subsection{Socked design}

The mesh model of the residual limb was modified using CAD modelling software packages Fusion 360 and Meshmixer to add and remove material to properly distribute load and prevent hot spots, Figure 5. Firstly, the mesh was cleaned and its volume was reduced by $3 \%$. This decision was made based on the viability of the tissue; viability being the quality and stage

Figure 4 Sound leg model and mirrored leg combined with residual limb used to size and align components for the generation of a monocoque design

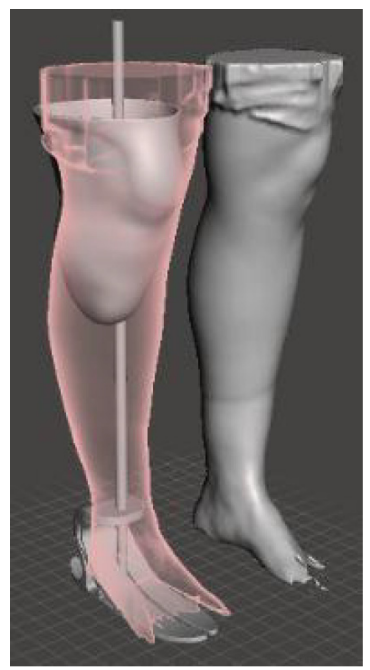

Figure 5 Design Process for the generation of a custom fit socket
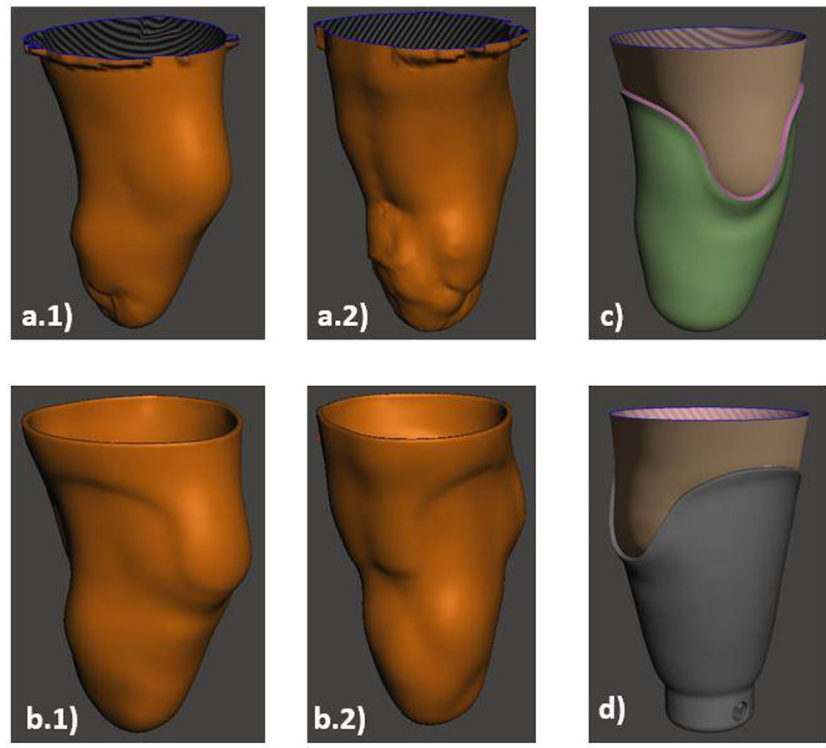

of the muscle tissue protecting bones and nerves. The determination of mesh reduction is, generally, that for an atrophied or more mature residual limb, the reduction is $1 \%-$ $3 \%$, whereas, for a residual limb that has more tissue and less atrophy, the reduction is $4 \%-5 \%$. In this work, the final volume reduction was determined by our collaborator, Jesus Mendoza, a certified prosthetist and orthotist (CPO). Next, a smoothing operation was used evenly to fill and smooth all scar tissue capture during the scanning process. In the A-P axis (anteriorposterior) we carved a $2 \mathrm{~cm}$ wide by $1 \mathrm{~cm}$ deep slot for the patellar tendon bar, which will be a primary load-bearing feature. We then compressed the medial-lateral proximal area by $2 \%$ to achieve a tight fit. Finally, socket trim lines were delineated to allow proper knee bending and mobility. This process will change from patient to patient and requires guidance from a CPO. A $4 \mathrm{~mm}$ thick shell was then extruded from the modified residual limb model's surface to create the socket.

\subsection{Pylon design}

The pylon was designed to be strong enough to satisfy the strength requirements in ISO standard 10328:2006. ISO 10328:2006 specifies loading and boundary conditions that prosthetic devices must fulfil. The pylon is expected to withstand a compressive proof load of $1,395 \mathrm{~N}$ and a maximum load of $3,220 \mathrm{~N}$. Additionally, the pylon is expected to withstand a maximum torque of $50 \mathrm{~N}-\mathrm{m}$. The solid-body produced in Rhinoceros 3D of the mirrored and segmented healthy leg was used to create the pylon. To reduce printing time and material cost, topology optimization (TO) software Inspire (Altair, MI, USA) was used to remove material and create an optimal structure. TO is a mathematical method that optimizes material layout within a given design space for a given set of loads, boundary conditions (BC) and constraints. Most TO formulations used today make use of finite element methods (FEM) to evaluate the design performance. The 
design is optimized using gradient-based mathematical programming algorithms such as the optimal criteria algorithm.

Model preparation for TO required the definition of design space, where the topology is optimized and BC regions, where loads and constraints are placed. The long dimension of the model was aligned with the $z$-axis. BC regions were defined as 0.1 millimetre thick $x-y$ slices at the top and bottom, with the remaining material as design space. $\mathrm{BC}$ regions were rigidly connected to the design space. As per ISO standard 10328:2006, $50 \mathrm{Nm}$ moment loads in $x, y$ and $z$ axes and a $3,220 \mathrm{~N}$ normal load along the $z$-axis were placed on the top $x-y$ $\mathrm{BC}$ region. The bottom $\mathrm{BC}$ region was fixed in all axes. The design space and BC regions were defined as Nylon Polyamide 12 , with elastic modulus, yield strength and Poisson's ratio set to $1.4 \mathrm{GPa}, 60 \mathrm{MPa}$ and 0.41 , respectively. Material properties used are based on filament manufacturer datasheets. Inspire only considers elastic behaviour, so material properties such as ultimate tensile strength, that operate in plastic deformation are not relevant. Topology optimization was performed with the objective of maximizing stiffness. Target mass reductions ranging from $30 \%-90 \%$ with $10 \%$ steps were run. Based on our requirement that segments be continuous, to enable FFF additive manufacturing, $70 \%$ was the optimal mass reduction target.

The resultant mesh was exported as an STL file, imported into the CAD software Meshmixer (Autodesk, CA) and smoothed and scaled to the correct dimensions. Correct dimensions and alignment were determined by superimposing the original sound leg mesh.

Loading and boundary conditions for the topology optimization study were extracted from the ISO standard 10328:2006 [25]. The generation of the new design allowed a perfect model to be used as a linking mechanism between the socket and foot without the need for third-party metallic components. The resulting bio-truss was used to connect the socket and foot into a monocoque design that can be fully printed in one piece. The resulting pylon structure can be seen in Figure 6.

\subsection{Ankle-foot complex design}

The first step towards the production of a functional foot prosthesis was the model acquisition. Once again, plane-cut operations were used to segment the foot section of the mirrored healthy leg scan model with the seven-foot isolated. A series of topology optimization studies, similar to the one presented in the prior section, was performed to reduce mass and maximize stiffness. Throughout topology optimization, simulations were performed to validate the optimized structures. Once an acceptable mesh was produced, it was exported as a .stl and imported into Fusion 360. A further modification to create attachment points and refine the external profile for matching desired shoes was deemed critical.

For this reason, a model was created manually using the mesh as a $3 \mathrm{D}$ stencil. This solid part was subsequently modified and simulated following ISO standard 10328:2006 [25]. The modelled geometry was $3 \mathrm{D}$ printed and tested but was ultimately limited in its functionality due to the numerous struts, as well as requiring a significant amount of time to model each iteration. For these reasons, a simplified model was created, which will be universally viable between patients/users,
Figure 6 (a) 3D Scan of the tibial region of the healthy leg. (b) Topology optimization of Pylon Region. (c) Smooth version of the optimized pylon

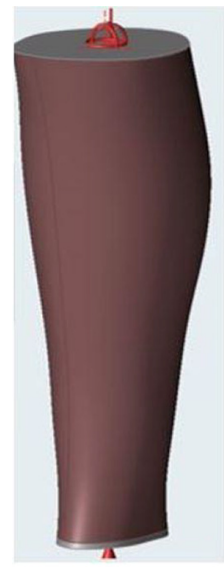

(a)

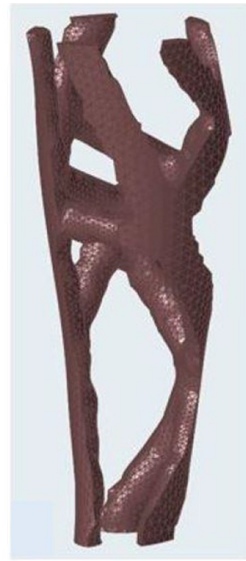

(b)

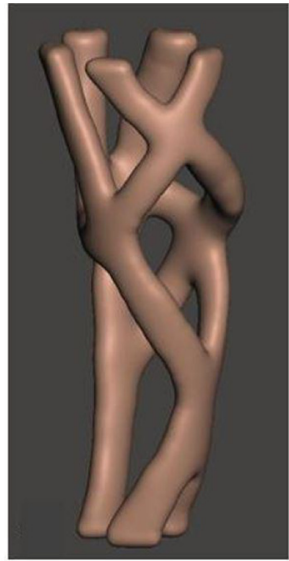

(c) easily tuned for the use-case, activity level and patient weight/ size, aesthetically pleasing, easily simulated and incorporates functional design elements to passively enable energy recapture and a smooth gait. To transition from the complicated, manually modelled and topology optimized geometry to the simplified model, several key design elements were identified and translated, as highlighted in Figure 7.

Finite element analysis (FEA) of foot - The below-knee prosthesis structural testing standard ISO 10328:2006 gives a comprehensive set of loading conditions that prosthetic devices must satisfy before being certified. The proof force is defined as the set of loading conditions where success is dependent on minimal permanent deformation. The proof force for activity level P5 at the heel and forefoot loading conditions for anklefoot devices and foot units in the static test procedure is $2,240 \mathrm{~N}$. The footing material was defined as isotropic Nylon PA-12, see Table 1 for mechanical properties. Contact surfaces were set to avoid surface penetration, as shown by dotted, curved lines in Figure 8. Forefoot and heel loading were

Figure 7 (a) Initial topology optimized foot design using the healthy leg mirrored model as reference geometry. (b) Modified design from the initial topology optimized version. (c) Important design elements identified on manually-modelled design. (d) Simplified model with translated design elements highlighted

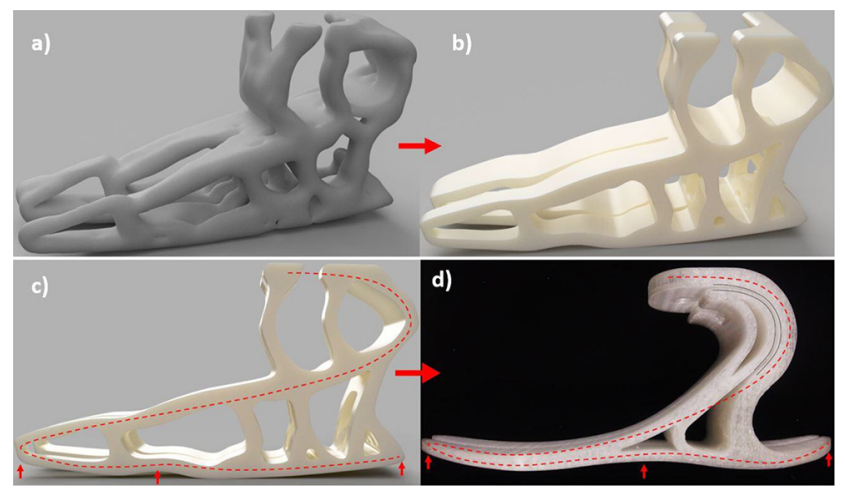


Table 1 Material properties for 3D printer filament [mcpp-3dp.com]. Standards followed are, density by ISO 1183, yield strength, elastic modulus and total elongation by ISO 527 and impact strength by Charpy notched testing at $23^{\circ} \mathrm{C}$ by ISO 179

\begin{tabular}{lccccc}
\hline Material & Density $\left(\mathbf{g} / \mathbf{c m}^{3}\right)$ & Yield strength $(\mathrm{MPa})$ & Elastic modulus $(\mathrm{GPa})$ & Total elongation $(\%)$ & Impact strength $\left(\mathrm{kJ} / \mathbf{m}^{2}\right)$ \\
\hline PLA & 1.24 & 69 & 3.14 & 4 & 3.4 \\
PETG & 1.27 & 50 & 2.02 & 23 & 8.1 \\
PA-12 & 1.02 & 60 & 1.40 & 250 & 14 \\
PC & 1.20 & 63 & 2.35 & 120 & 60 \\
\hline
\end{tabular}

performed as specified by ISO10328 with the top surface of the ankle fixed, in Figure 8. One additional loading condition was used to test for proper forward bending resistance at the foot/ ankle-pylon connection. The two ground contact points, B in Figure 8 , on the bottom of the foot were fixed. The same proof force of 2,240 $\mathrm{N}$ was applied in the u-axis, synonymous with the TKA line.

\subsection{Digital alignment, integration and monocoque design}

The first prototype created by the team was a multi-component device that had to be connected and aligned using metal thirdparty components. Because of the help of our CPO collaborators, Herb Barrack and Jesus Mendoza, we were able to get access to medical graded prosthetic components.

Figure 8 Foot unit loading and boundary conditions diagram

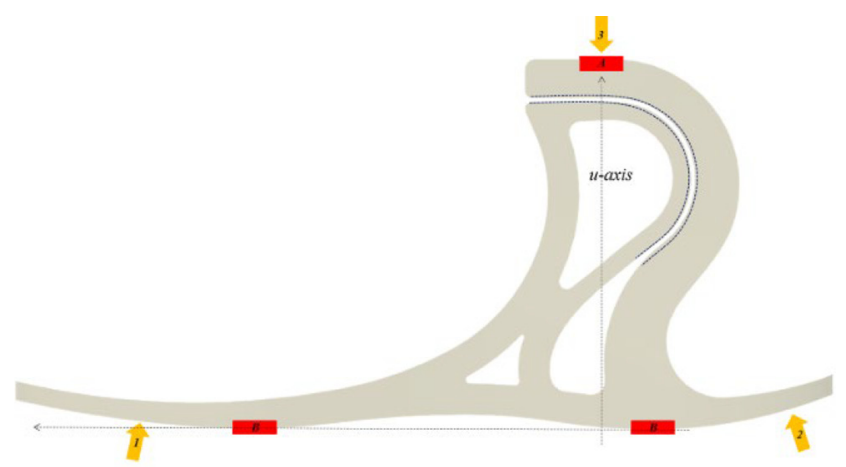

Figure 9 Components used as connections in standard designs

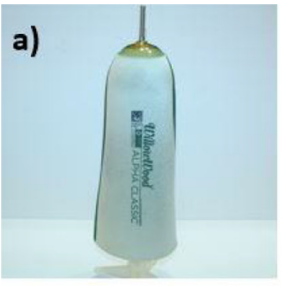

Figure 9 showcases all the different elements required for the full assembly of our first prosthetic leg prototype. Firstly, a custom-made silicone liner:

- with an embedded titanium pin rod

- had to be ordered and was manufactured by WillowWood, $\mathrm{OH}$.

The liner helps to improve the fit between the damaged limb and the $3 \mathrm{D}$ printed custom socket. Also, it serves as a cushion and damper to improve patient comfort. WillowWood also provided us with a shuttle lock mechanism that had to be embedded into our 3D printed socket so the liner and the socket can connect securely. The next stage was to connect the pylon to the socket and foot. For this, we were provided several modular endoskeleton components also by WillowWood and can be seen in Figure 9(e). The first components were a socket attachment plate connected to a titanium 4-hole pyramid adapter that connected to a titanium pylon adapter which further connects with a pipe embedded in the design of the topology optimized pylon. Next, we connected the pylon with the foot also using a titanium 4-hole pyramid adapter and a titanium pylon adapter. It is important to notice that the pyramid and pylon adapters served not only as structural attachment elements but also as alignment devices that allowed the prosthetists to properly align the prosthesis. The final external component used in this first prototype series was a silicon cover that helps the foot to fit shoes and improve aesthetics when desired. All components were assembled as represented in Figure 10(f).

The components presented in the previous paragraph are industry standards that CPOs rely on to assemble prosthetic devices. It is worth noting that although these components are

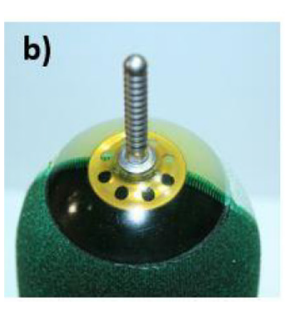

c)

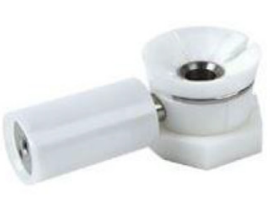

d)
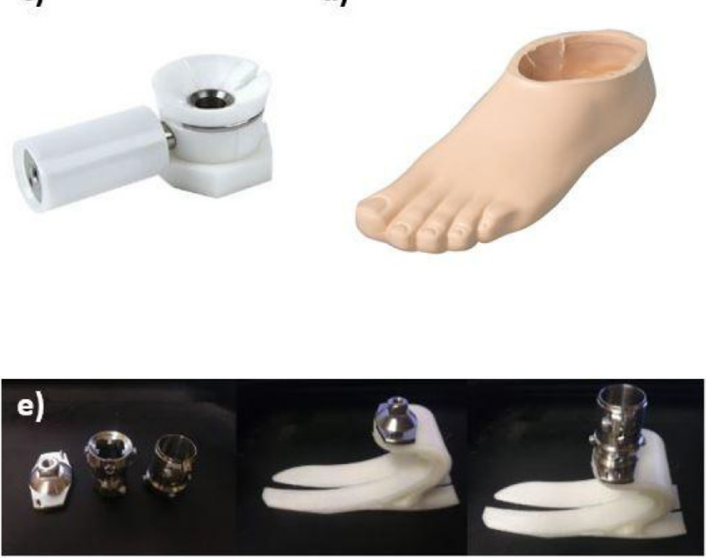

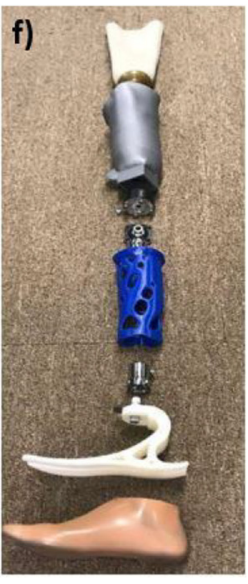


Figure 10 (a) Using the healthy leg scan and its mirrored model we were able to properly scale and align all components so a ready to use monocoque version could be printed. (b) Side view of final monocoque design

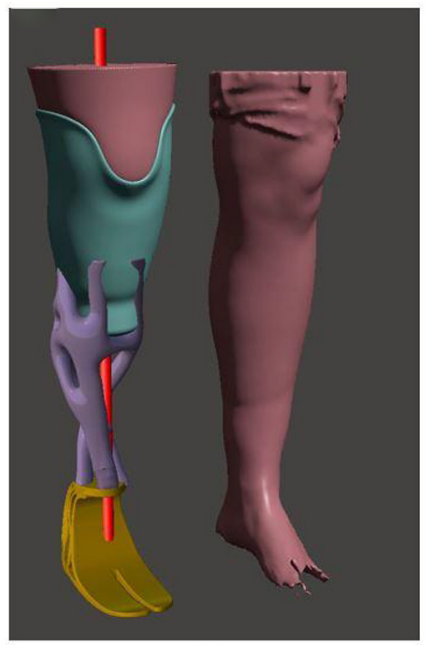

(a)

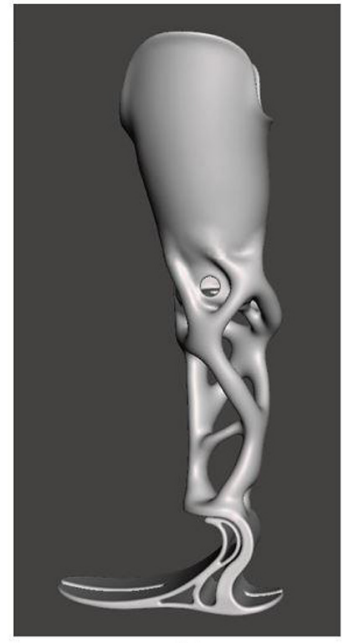

(b) reliable, they are also expensive and require expertise to properly align. With the goal of making our prosthetic devices accessible worldwide, we focused our design process around minimizing third-party components. Using digital design and additive manufacturing, we were able to align and connect our socket, pylon and foot digitally to produce a monocoque prosthesis device Figure 10. The monocoque was designed using the mesh editing software Meshmixer. For alignment, we used the mirrored healthy leg scan as a guide to place and connect the three components. We draw a cylinder that runs through the middle of the mirrored leg and is aligned by the knee and ankle positions, Figure 10(a). The resulting monocoque design, Figure 10(b), was fully printed in one piece. The only commercial component used was the shuttle lock which was designed to perfectly fit and secure in the $3 \mathrm{D}$ printed monocoque.

\subsection{Additive manufacturing}

In this study, we used a series of fused filament fabrication (FFF) machines such as the 3DP 300 Series Workbench (3D Platform, IL), a double nozzle large scale format 3D printer with a print volume of $1,000 \times 1,000 \times 700 \mathrm{~mm}$. The selection of FFF as the 3D printing technique was made because of the cost-effectiveness of the machines, materials and the process itself. It also offers a broad range of materials to work with ranging from prototyping materials such as PLA to ready to wear materials like Nylon Polyamide 12 (PA-12). It is also the most environmentally friendly and can be performed in an office or hospital environment. The 3DP large factor equipment was vital in the manufacturing of the monocoque prosthetic, as it allowed us to print tall objects in one piece. We also made use of smaller 3D printers such as the LulzBot TAZ 6 for the generation of individual components. Maximum nozzle temperatures between $250^{\circ} \mathrm{C}$ and $300^{\circ} \mathrm{C}$ enable the use of the most available filament types.
The large selection of thermoplastic filament available for the FFF process allowed us to select our feedstock material with our specific use-cases in mind. Group one materials for prototyping, alignment and diagnostic test fitting, included low-cost materials with high printing performance and lower mechanical properties. Group two materials, for load-testing and end-use parts, were selected primarily for their mechanical properties. The materials selected for group one included polylactic acid (PLA) and polyethylene glycol (PET-G). Lower printing temperatures between $190^{\circ} \mathrm{C}$ and $240^{\circ} \mathrm{C}$ and small coefficients of thermal expansion reduce bed de-adhesion, delamination of layers and bulk warping in parts printed with each of these filaments. Group two materials include nylon (PA-12), which have higher mechanical properties but require more challenging printing conditions.

PA-12 is hydrophilic and must be dried before printing and sealed in a container with desiccant during printing. This material requires printing temperatures between $260^{\circ} \mathrm{C}$ and $280^{\circ} \mathrm{C}$, build platform temperatures between $80^{\circ} \mathrm{C}$ and $100^{\circ} \mathrm{C}$ and chamber temperatures higher than $40^{\circ} \mathrm{C}$. Material properties used for topology optimization, FEA and material selection are presented in Table 1 (Anon, 2021). The lower modulus and higher elongation of PA-12 made it a suitable candidate for the foot and ankle, which needs to be flexible and have high fatigue resistance for the dynamic gait cycle. PLA was rejected due to its low total elongation and impact strength. The socket and pylon require strong and stiff behaviour, which made PETG the better candidate. Further testing of the pylon and socket may identify a need for higher toughness, but for this study, PETG and PA-12 filaments were used to print loadbearing, end-use parts.

We used additive manufacturing techniques to print fullscale models that were used to test the fit, comfort and mechanical bearing capacity under a controlled load environment, as well as active patient loading. 3D printing not only allowed us to fabricate custom parts on-demand in a costeffective manner but also allowed us to quickly turn digital models into functional prototypes; a key factor in enabling an iterative design process. The process to $3 \mathrm{D}$ print any model is as follows:

First, a 3D CAD model is exported as a closed STL file. The file is then imported into slicer software. In our case, we used Simplify3D (Cincinnati, OH). In Simplify, the model was placed in the desired print bed position and printing parameters such as speed, temperature and supports were defined. The slicer then generates a G-code file, which gives layer by layer information to the $3 \mathrm{D}$ printer. The slicer then generates a Gcode file, which gives layer by layer information to the $3 \mathrm{D}$ printer. The printer bed and nozzle temperature were set, as well as preparing the printing bed with glue to improve adhesion. The printer was started and monitored for any failures during the printing process. Finally, the printed model was then removed from the bed and support structures were removed with pliers.

Using this manufacturing method, we were able to successfully $3 \mathrm{D}$ print multiple sockets, pylons, feet as seen in Figure 11 and monocoque designs in Figure 16. The result is a reliable, fast and cost-effective way to manufacture custom prosthetic devices, which will enable us in our goal to bring reliable custom prostheses to those in need. 
Figure 11 Final version of 3D printed

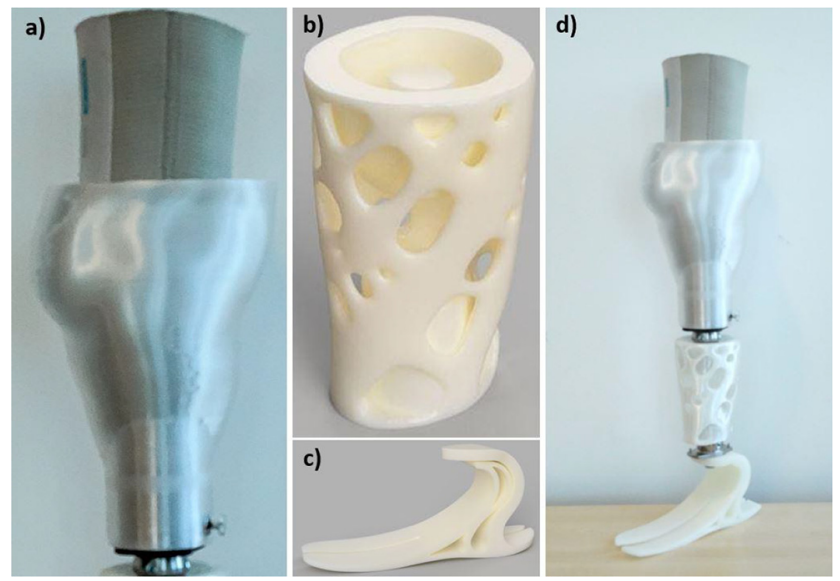

\section{Results and discussion}

\subsection{Three-dimensional imaging}

After successfully reconstructing the patient's residual limb morphology with both Photogrammetry and Laser Scanning techniques we compared the two models using a 3D point cloud and mesh processing software such as CloudCompare (CloudCompare, France). To compare the accuracy and quality of the photogrammetry scan we imported both models in .xyz format, aligned them using reference points such as the tip of the limb or the kneecap, calculated surface normal on both models and performed a cloud-to-cloud distance operation. The results show a maximum local difference of $1.2 \mathrm{~mm}$ and a minimum local difference of $2.0 \mathrm{~mm}$. The resulting colour image, Figure 12, illustrates the difference between the two models. These results allowed us to confirm the quality of the photogrammetry model and quantify the quality of photogrammetry scanderived models prior to $3 \mathrm{D}$ printing and patient trials. The team was able to lock down a repeatable procedure to convert photogrammetry derived model into a functional, anatomically correct custom-made 3D printed socket. Our test patient, D.N., was delighted with the performance and comfortability of the $3 \mathrm{D}$ printed device.

\subsection{Pylon}

We used FEA to validate the safeness of the topology optimized pylon section. We applied the ISO 10328:2006 loads of maximum compression $(3,220 \mathrm{~N})$ and maximum torque (50 $\mathrm{Nm}$ ) on the top of the model and fixed the bottom surface for translation and rotation. We defined boundary conditions as fixed on the bottom face and loads applied on the top face, the material used was isotropic Nylon Polyamide 12 and the mesh was created and refined by the solver in SolidWorks. The results, Figure 13, shows the Von Mises stress $\left(\mathrm{N} / \mathrm{m}^{2}\right)$ regions under ISO standard loads. Maximum stress can be found on the frontal-bottom section and the back bottom section, both regions can be seen in red colour in Figure 13. The maximum stress of $16 \mathrm{MPa}$ is derived from the pylon simulation, which, when compared to its yield strength of $60 \mathrm{MPa}$ for PA-12, results in a satisfactory factor of safety of 3.75.

\subsection{Ankle-foot complex}

In this study, the proof force, as defined by the ISO 10328:2006, was analysed using nonlinear static stress FEA simulations at heel, forefoot and ankle locations to verify plastic yielding and elastic deformation values stay within set boundaries. Material used for these simulations was isotropic Nylon Polyamide 12. In the forefoot loading condition:

- shown in Figure 14, a maximum deflection of $62.08 \mathrm{~mm}$ occurs at the toes and the maximum Von Mises stress is 95.61 $\mathrm{MPa}$. In the heel loading condition,

- shown in Figure 14, a maximum deflection of $9.06 \mathrm{~mm}$ occurs at the toes and the maximum Von Mises stress is $43.06 \mathrm{MPa}$. A maximum deflection of $3.21 \mathrm{~mm}$ in the negative u-direction occurred at the ankle joint and maximum Von Mises stress of 31.75 was calculated for condition,

- in Figure 14 transition and is enabled due to the disconnected-surface foot design.

Normal loading along the $\mathrm{u}$-axis resulted in a small deflection that was within acceptable limits for acute hip height misalignment, as

Figure 12 Using CloudCompare we were able to quantify the difference between the photogrammetry model and the laser scanner model. Distances are displayed as a colour map with units in $\mathrm{mm}$

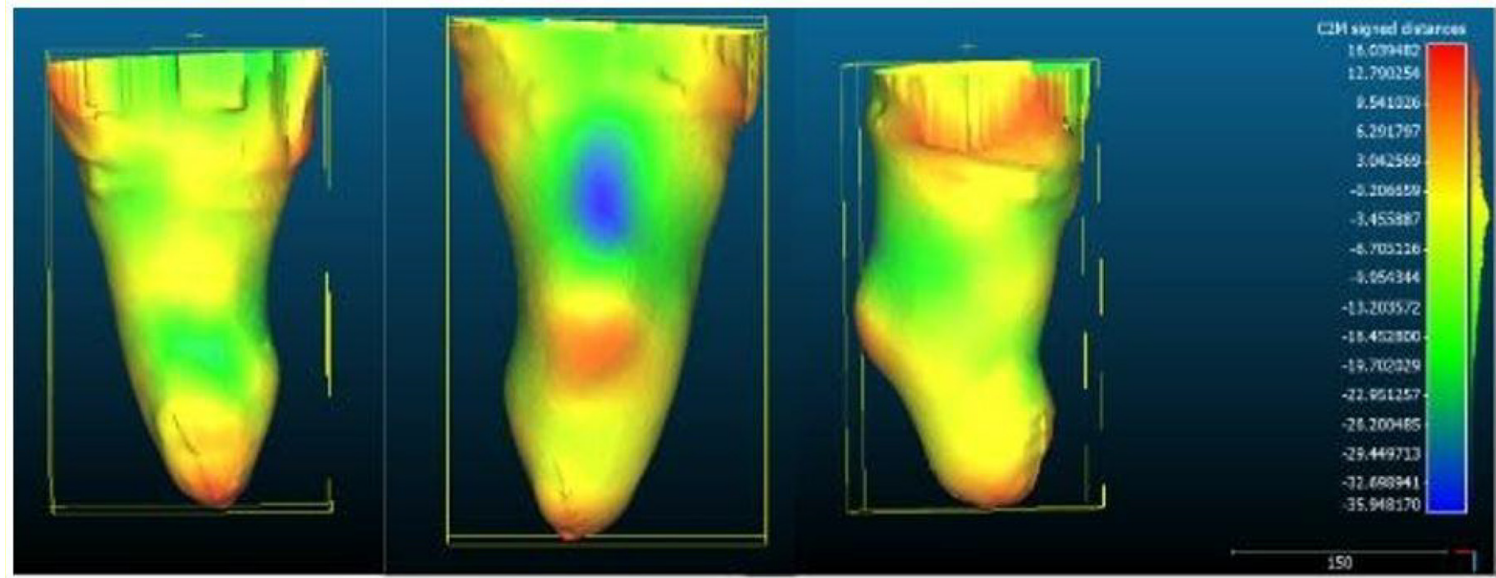


Figure 13 Finite element analysis of Pylon under ISO standard 10328:2006 compressive and torsional loads. The maximum stress of 16 MPa was found

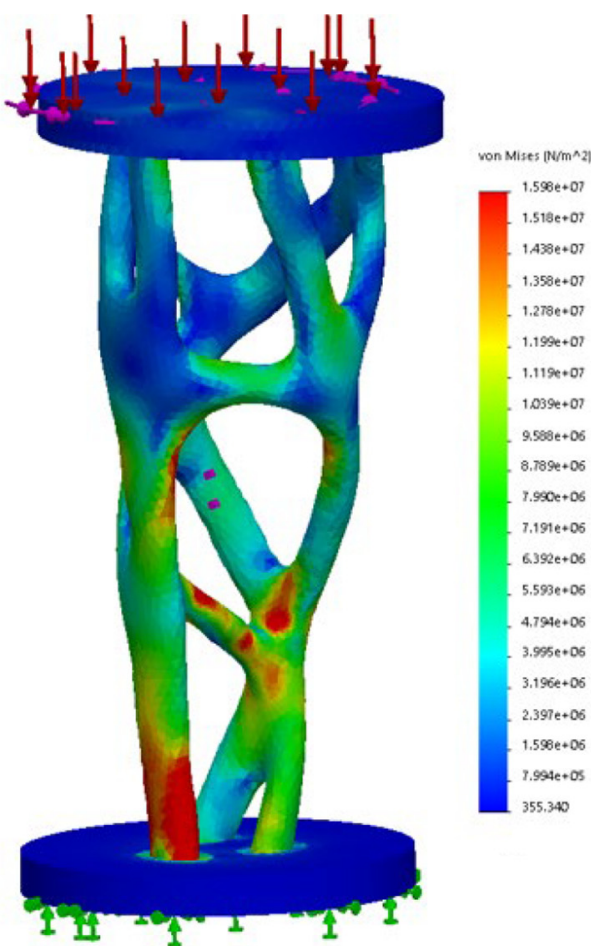

(a)

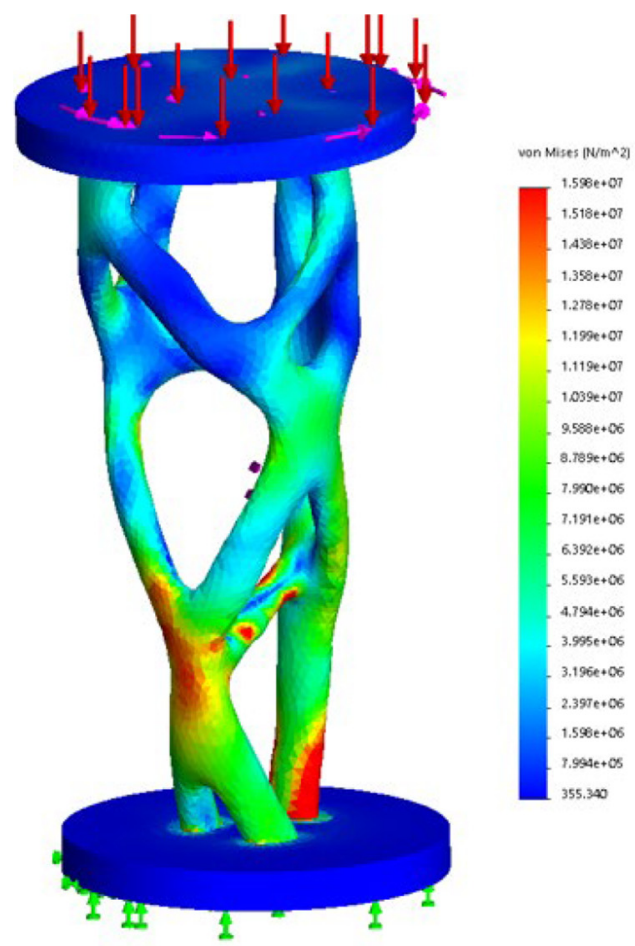

(b)

Notes: (a) Anterior view; (b) posterior view

validated by CPO Herb Barrack. The mid-stance to forefoot-liftoff transition is aided by the stored spring energy from the $62.08 \mathrm{~mm}$ deflection, resulting in lower metabolic costs during the gait cycle.

\subsection{Monocoque}

One of the challenges of this study was to quantify and validate the advantages of 3D printable transtibial prosthetic monocoques over traditional prosthetic legs. To accomplish this, we decided to break it down into metrics. The metrics used were amputee feedback, alignment success, weight comparison, cost analysis and time of design and manufacture.

\subsubsection{Socket comfort}

Using digital design enabled the modification of the socket scan for the addition of load-bearing regions for the patella tendon and developing proper spacing at sensitive areas such as bony protrusions. The feedback from our patient, D.N., was the best way for us to validate the comfort and fit of the $3 \mathrm{D}$ printed socket. "I am amazed at how I can just take something that did not exist $12 \mathrm{~h}$ ago and just put it on and walk away. The comfort and fit are beyond impressive. The process is so customized that it allows for maximum comfort". The positive feedback from our patient and her doctor indicates the success of our digital design workflow.

\subsubsection{Alignment trochanter-knee-ankle line}

Correct alignment is essential for both short-term comfort and the long-term health of patients. Even a small misalignment, which can be difficult for an untrained eye to see, can cause a host of biomechanical issues, including complications to the

Figure 14 Foot unit FEA results showing deformation in (A) forefoot, $(B)$ heel and $(C)$ u-axis loading conditions

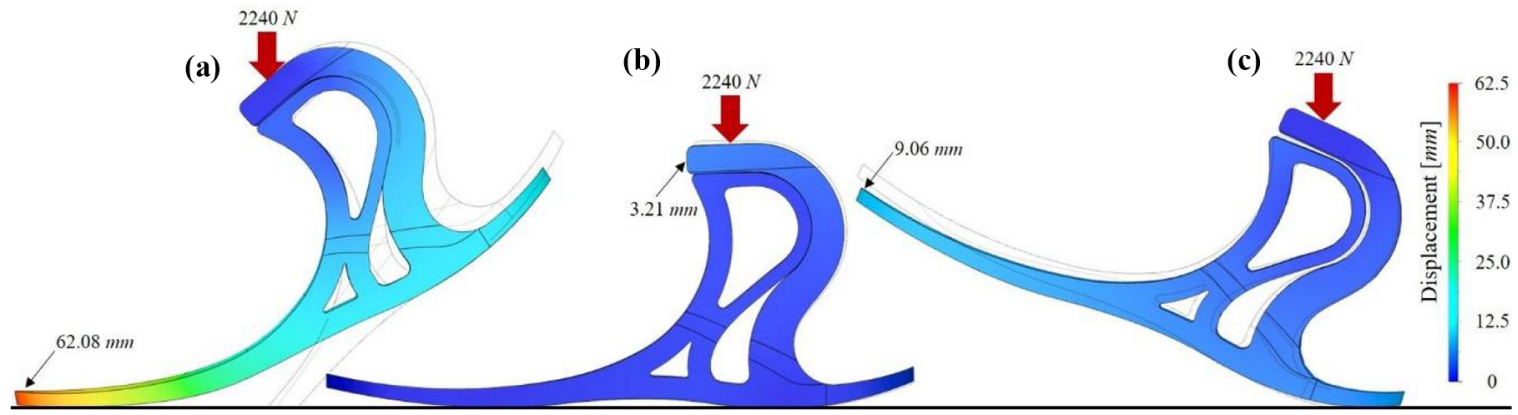


wearer's knees, hips and spine. To validate the alignment of the resulting leg, we relied on Certified Prosthetist/Orthotist Herb Barrack for his expertise. Anterior and lateral pictures were also taken of patient D.N. standing tall wearing our monocoque device, Figure 15. For proper TKA alignment, the trochanter, knee and ankle points must fall along a vertical line during standing position, Figure 15(b).

\subsubsection{Cost analysis}

The principal motivation behind this work was solving the worldwide prosthesis accessibility crisis. The expensive nature of prosthetic devices drives this crisis, where 38 million people worldwide do not have access to any form of a prosthetic device. The price of a new prosthetic leg can cost anywhere from $\$ 5,000$ to $\$ 50,000$. However, even the most expensive prosthetic limbs are built to withstand only three to five years of wear and tear, meaning they will need to be replaced throughout a lifetime and they are not a one-time cost. Usually, the total cost of service for a socket will be billed to an insurance company or Medicare at $\$ 10,000$ for an above-the-knee amputee and $\$ 7,000$ for a below-the-knee amputee, on average. A typical passive foot in the US costs $\$ 1,000$ to $\$ 10,000$ and is made from carbon fibre. Other component costs range from $\$ 50$ to $\$ 500$ as is the case for lock mechanisms, foot shells and custom liners. The full cost analysis can be found in the list below. Using this list, we were able to compare the full cost of materials for making a traditional prosthetic versus our fully $3 \mathrm{D}$ printed monocoque prosthesis. It should be noted that this comparison focuses on only the cost of materials. Through our analysis, we can report a material cost reduction of $95 \%$ for our $3 \mathrm{D}$ printed prosthetic device compared with traditional prostheses.

Traditional cost of the transtibial prosthetic leg by components and materials: Custom Made Socket $\$ 7,000.00$, Custom Liner $\$ 500.00$, Titanium Pylon $\$ 150.00$, Ottobock

Figure 15 Trochanter-knee-ankle (TKA) alignment shown for patient D.N. Wearing the 3D printed Monocoque prosthesis

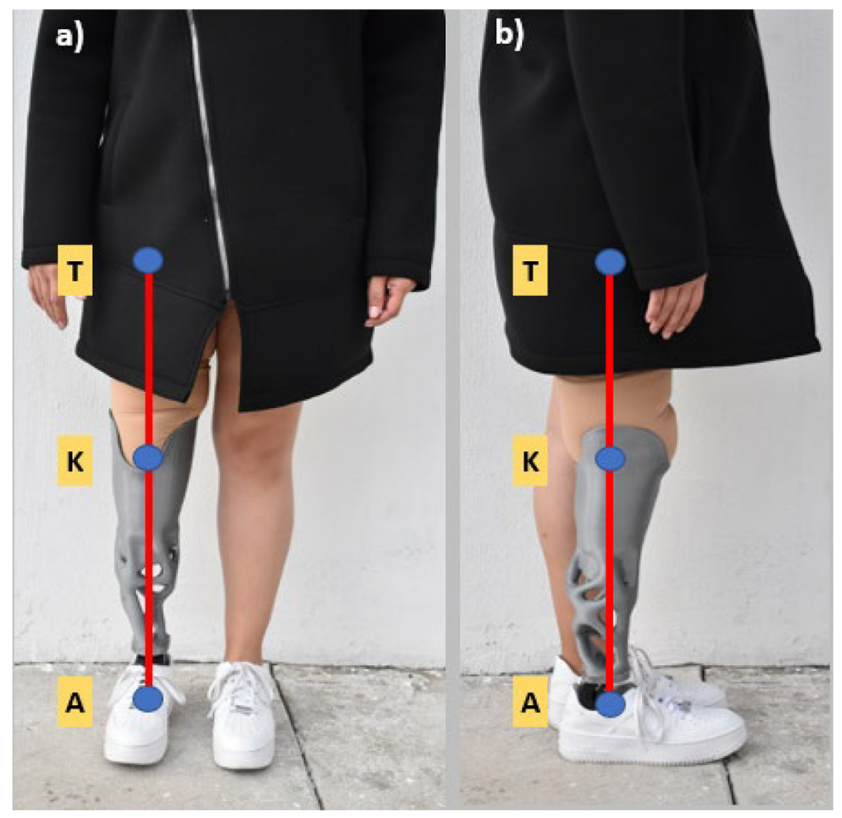

Triton Foot $\$ 2,000.00$, Foot Shell $\$ 150.00,4$-hole Safety Lock w pin $\$ 200.00$, Stainless Steel 4-hole Plate $\$ 50.00$, Titanium 4-hole Male Pyramid \$60.00, Titanium 4-hole Female Pyramid \$60.00, Titanium Tube Clamp (2x) \$70.00 each.

Total traditional cost: $\$ 10,310.00$.

LIMBER 3D printed monocoque by components and materials: PA-12 Filament $1 \mathrm{Kg}$ (Monocoque): \$140.00, Flexible Resin 1 L(Liner) \$65.00, hole Safety Lock w pin $\$ 200.00$, Foot Shell $\$ 150.00$.

Total LIMBER monocoque cost: $\$ 550.00$.

\subsubsection{Weight comparison}

In addition to alignment and cost, we compared the weight of a traditional prosthetic against our device. For the conventional prosthetic, we weighed a fully Assembly leg with its three main components (Socket, pylon and foot) and all necessary extra components (plates, pyramid connectors, etc.). The final weight of patient D.N.'s traditional prosthetic was 4 pounds, whilst the LIMBER device was only $1.8 \mathrm{lbs}$; a 55\% weight reduction. Patient D.N. commented on the lighter feel of the 3D printed prosthesis and noted it increased her comfort and decreased the effort required to walk.

\subsubsection{Time of design and manufacture}

The final metric used in the study to quantify the success of the LIMBER monocoque was to derive the time it took to design and manufacture compared to a traditional prosthetic. For a conventional prosthetic, various steps need to be taken before we can even start creating the final prosthetic. Firstly, the CPO needs to measure the patients remaining and healthy limbs manually. These measurements will help the CPO size the components required for the final assembly. Next, the CPO must make a mould of the residual limb, which is used to generate the loadbearing regions and ears of the socket. This mould serves as a base for the lamination of CFRP or other composite materials. Once the resin cures, the socket is de-moulded, trimmed and surface finished. Then, they attach the plates and locking mechanisms to the socket and finally assemble all components. Due to the problematic craftsmanship and multiple required patient visits, this process takes an average of 14 days from start to finish. For the Limber Monocoque, there are three main steps required to produce a custom prosthesis. Firstly, surface data is acquired using a laser scanner or camera and a model is reconstructed from the collected point cloud. Secondly, the socket is created and the pylon and foot are sized according to using the healthy leg scan. Thirdly, the digitally designed prosthesis is sliced into G-code and simply sent to the printer. Altogether, it takes around $16 \mathrm{~h}$ to go from scan to final printed monocoque, with no additional patient visits required. A breakdown of time can be found on the list below:

Traditional transtibial prosthetic leg by design and manufacture time: measurements (1 Day), mould creation (3 Days), socket creation (6 Days), assembly and alignment (4 Days). Total traditional time: 14 Days.

LIMBER monocoque design and manufacture time: data acquisition $(1 \mathrm{~h})$, design time $(3 \mathrm{~h})$, printing time $(12 \mathrm{~h})$. Total limber monocoque time: $16 \mathrm{~h}$.

\section{Conclusion}

The successful fabrication of a functional monocoque prosthesis through $3 \mathrm{D}$ printing indicates our workflow may be a 
solution to the worldwide accessibility crisis. The digital workflow developed in this work offers great potential for providing prosthetic devices to rural communities, which lack access to skilled prosthetic physicians or where high-tech, medical imaging devices such as CT scanners are not available or cost-prohibitive. The work of this paper presented the design and manufacturing of a $3 \mathrm{D}$ printed transtibial prosthesis, including socket, pylon, foot and a monocoque design. Our goal was to demonstrate the ability to create a comfortable, functional and cheap prosthesis through digital design and manufacturing techniques. We quantified the benefits of our workflow using several metrics. We found that using our workflow together with 3D printing, we can create custom monocoque prostheses (Figure 16). These prostheses are comfortable, functional and properly aligned. In comparison with traditional prosthetic devices, we lowered the average cost, weight and time of production by $95 \%, 55 \%$ and $95 \%$, respectively. For future work, to ensure this method is suitable for other patients, we will perform benchmarking tests with other patients and mechanical tests to compare the maximum load capacity and cyclic performance of our monocoque $3 \mathrm{D}$ printed design, using a range of different engineering-grade $3 \mathrm{D}$ printable material filaments, to a traditional prosthesis. Once we validate the response of the $3 \mathrm{D}$ printed monocoque by testing we will derive orthotropic material properties, doing a series of coupon tests and use these in our future FEA

Figure 16 Final version of full-scale LIMBER 3D printed monocoque prosthesis

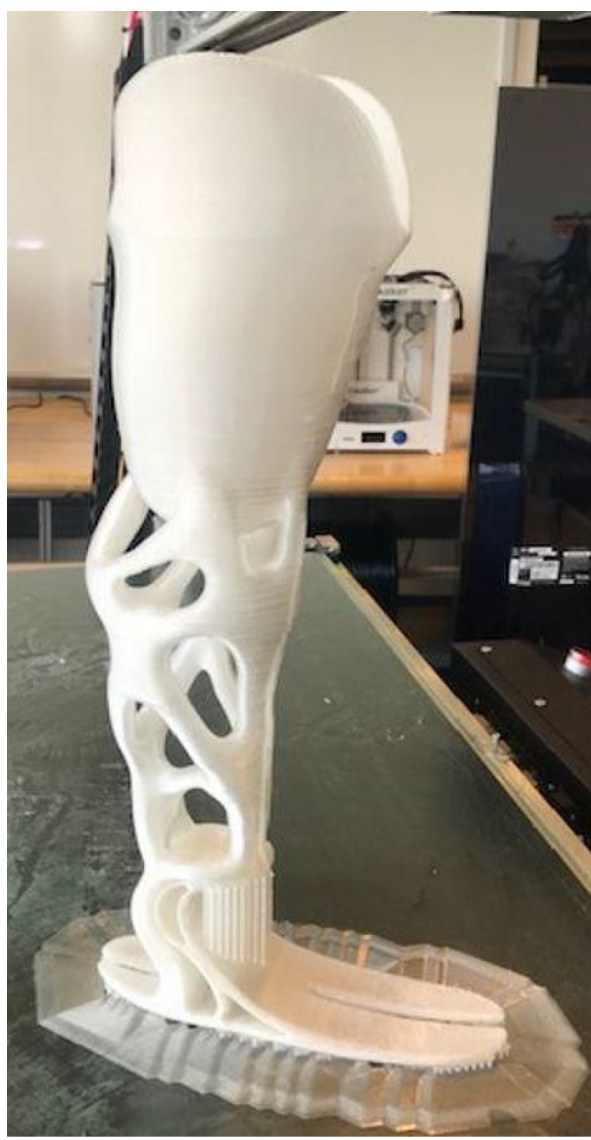

simulations. We will also conduct gate analysis using a gate lab to validate the alignment and performance of the monocoque during active use.

This novel digital design and manufacturing workflow has the potential to democratize and globally proliferate access to prosthetic devices, which restore the patient's mobility, quality of life and health. LIMBER's toolbox can reach places where proper prosthetic and orthotic care is not available. The digital workflow reduces the cost of making custom devices by an order of magnitude, enabling broader reach, faster access and improved comfort. Timely access is critically important during the early recovery period when residual limbs change in shape due to atrophy and scar tissue. In particular, for children who grow quickly and need new devices every few months or years, timely access is both physically and psychologically important.

\section{Acknowledgments}

We want to sincerely thank Diana Novitchenko, who courageously volunteered as our first patient and allowed us to scan her legs and experiment in the creation of a custom 3D printable prosthetic device. We hope this work allows us to reach many more like Diana, and especially those who do not have access to any kind of prosthetic device. We would also like to thank the ABI Prosthetic and Orthotic team, who introduced us to Diana and offered their incredible knowledge and expertise regarding creating prosthetic devices. Their help and participation in this project were of essential importance, and the final results of the study could not have been achieved without their experience and support. We want to give special thanks to the CHEI and UCSD Drone Lab team, together with the group of undergraduate and graduate engineering students (Gokul S Nair, Harleen Singh, Zach Pribyl, Patricia Castillo, Abel John Raymer, Matthew Ferrari, Victor Bourgin) for their effort and time spent on this project. A special thanks go to Benito Sweeney for creating the link between our lab and the ABI Prosthetic and Orthotic team. The collaboration between our multidisciplinary team made this exciting project possible.

\section{Funding}

This publication is based on work supported by the US Army Corps of Engineers under research Cooperative Agreement W912HZ-17-2-0024, National Institute of Standards and Technology Award \#70NANB17H211, as well as National Science Foundation award \#CNS-1338192, MRI: Development of Advanced Visualization Instrumentation for the Collaborative Exploration of Big Data.

\section{Author contributions}

Conceptualization, Luca De Vivo, Joshua Pelz, Herb Barrack and Falko Kuester; Data curation, Luca De Vivo and Jesus Mendoza; Methodology, Luca De Vivo, Joshua Pelz, Herb Barrack and Falko Kuester; Project administration, Falko Kuester; Resources, Herb Barrack and Falko Kuester; Writing - original draft, Luca De Vivo; Writing - review \& editing, Luca De Vivo, Joshua Pelz, Herb Barrack and Falko Kuester. 


\section{References}

Al Muderis, M., Lu, W. and Li, J.J. (2017), “Osseointegrated prosthetic limb for the treatment of lower limb amputations", Der Unfallchirurg, Vol. 120 No. 4, pp. 306-311.

Anon (2021), "MCPP 3DP - MCPP Netherlands BV", [online], available at: www.mcpp-3dp.com

Bregler, C., Hertzmann, A. and Biermann, H. (2000), "Structure from motion without correspondence", Proceedings IEEE Conference on Computer Vision and Pattern Recognition.

LeMoyne, R. (2016), "Advances for prosthetic technology", [online], Springer Japan, Tokyo, available at: https:/link. springer.com/book/10.1007\%2F978-4-431-55816-3 (accessed 9 Jun. 2021).

LeMoyne, R. (2016), "Passive transtibial prosthesis and associated prosthetic components", In Advances for Prosthetic Technology, Springer, Tokyo, pp. 59-68.

Pirouzi, G., Abu Osman, N.A., Eshraghi, A., Ali, S., Gholizadeh, H. and Wan Abas, W.A.B. (2014), "Review of the socket design and interface pressure measurement for transtibial prosthesis", The Scientific World fournal, Vol. 2014.
Prosthetic and Orthotic Care (2021), "Transtibial prosthetic leg", [online], available at: www.pandocare.com/transtibialprosthetics/

Schall, S.R. and Slemker, T.C. (1998), Prosthetic Design Inc, 1998. Method for fabricating a prosthetic limb socket. U.S. Patent 5,824,111.

Schuch, C.M. and Pritham, C.H. (1994), "International standards organization terminology: application to prosthetics and orthotics", FPO fournal of Prosthetics and Orthotics, Vol. 6 No. 1, pp. 29-33.

Sengeh, D.M. and Herr, H. (2013), "A variable-impedance prosthetic socket for a transtibial amputee designed from magnetic resonance imaging data", FPO: Fournal of Prosthetics and Orthotics, Vol. 25 No. 3, pp. 129-137.

Strait, E. (2006), "Prosthetics in developing countries", Prosthetic Resident, Vol. 1, pp. 1-3.

Ziegler-Graham, K., MacKenzie, E.J., Ephraim, P.L., Travison, T.G. and Brookmeyer, R. (2008), "Estimating the prevalence of limb loss in the United States: 2005 to 2050", Archives of Physical Medicine and Rehabilitation, Vol. 89 No. 3, pp. 422-429, [online], available at: www.sciencedirect.com/science/article/pii/ S0003999307017480 (accessed 17 April 2019). 


\section{Appendix}

The appendix is a supplementary file describing in detail the LIMBER workflow. This file explains the tools and software's used and step by step instructions on how to perform the LIMBER process from scanning, to processing, design and manufacturing. It is important for the reader to be aware that a Certified Prosthetist and Orthotist should ideally perform the activities or at the very least supervise each part of the process. This is a workflow that proved to deliver a working prototype of a below-knee prosthetic leg, but further testing and evaluations are necessary to certify this device and offer it to patients [ISO 10328:2016].

International Organization for Standardization. (2016). Occupational health and safety management systemsRequirements with guidance for use (ISO/DIS Standard No. 45001). Retrieved from www.iso.org/iso/catalogue_detail? csnumber $=63787$

\section{Corresponding author}

Luca Gabriele De Vivo Nicoloso can be contacted at: ldevivo@eng.ucsd.edu

Figure A1 LIMBER prostheses digital workflow

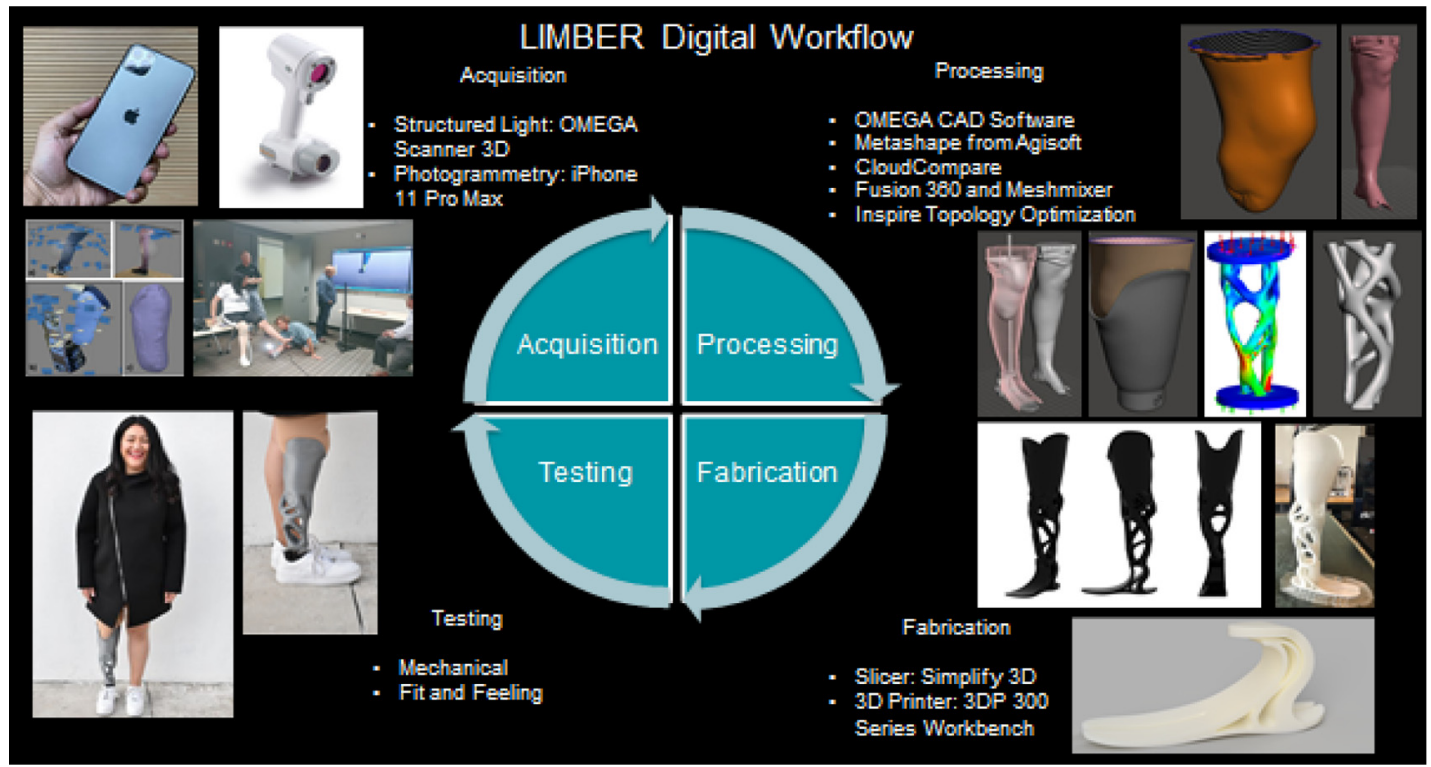

For instructions on how to order reprints of this article, please visit our website:

www.emeraldgrouppublishing.com/licensing/reprints.htm

Or contact us for further details: permissions@emeraldinsight.com 